\title{
Development of Small-Molecule Cyclin D1-Ablative Agents
}

Jui-Wen Huang, ${ }^{1}$ Chung-Wai Shiau, ${ }^{1}$ Jian Yang, ${ }^{1}$ Da-Sheng Wang, ${ }^{1}$ Ching-Yu Chen, ${ }^{2}$ and Ching-Shih Chen $^{1 *}$

${ }^{1}$ Division of Medicinal Chemistry and Pharmacognosy, College of Pharmacy, The Ohio State University, Columbus, $\mathrm{OH} 43210$

${ }^{2}$ Department of Family Medicine, College of Medicine, National Taiwan University, and The Gerontology Research Division, The National Health Research Institutes, Taipei,

Taiwan

\section{Supporting Information}

Elemental analyses data

\begin{tabular}{|c|c|c|c|c|c|c|c|}
\hline \multirow{2}{*}{ Entry } & \multirow{2}{*}{ Formula } & \multicolumn{3}{|c|}{ Anal. Calcd. } & \multicolumn{3}{c|}{ Found } \\
\cline { 3 - 8 } & & $\mathrm{C}(\%)$ & $\mathrm{H} \mathrm{( \% )}$ & $\mathrm{N}(\%)$ & $\mathrm{C}(\%)$ & $\mathrm{H}(\%)$ & $\mathrm{N}(\%)$ \\
\hline $\mathbf{1}$ & $\mathrm{C}_{27} \mathrm{H}_{29} \mathrm{NO}_{5} \mathrm{~S}$ & 67.62 & 6.09 & 16.68 & 67.37 & 6.07 & 17.01 \\
\hline $\mathbf{2}$ & $\mathrm{C}_{29} \mathrm{H}_{33} \mathrm{NO}_{5} \mathrm{~S}$ & 68.61 & 6.55 & 15.76 & 68.54 & 6.33 & 15.40 \\
\hline $\mathbf{3}$ & $\mathrm{C}_{31} \mathrm{H}_{31} \mathrm{NO}_{5} \mathrm{~S}$ & 70.30 & 5.90 & 15.10 & 69.98 & 5.78 & 14.95 \\
\hline $\mathbf{8}$ & $\mathrm{C}_{28} \mathrm{H}_{31} \mathrm{NO}_{6} \mathrm{~S}$ & 65.99 & 6.13 & 18.84 & 65.84 & 6.16 & 18.61 \\
\hline $\mathbf{9}$ & $\mathrm{C}_{28} \mathrm{H}_{31} \mathrm{NO}_{5} \mathrm{~S}$ & 68.13 & 6.33 & 16.21 & 68.45 & 6.53 & 16.05 \\
\hline $\mathbf{1 2}$ & $\mathrm{C}_{29} \mathrm{H}_{32} \mathrm{BrNO}_{5} \mathrm{~S}$ & 59.38 & 5.50 & 13.64 & 59.72 & 5.77 & 13.70 \\
\hline$(S)-8$ & $\mathrm{C}_{28} \mathrm{H}_{31} \mathrm{NO}_{6} \mathrm{~S}$ & 65.99 & 6.13 & 18.84 & 66.15 & 6.09 & 18.96 \\
\hline$(R)-8$ & $\mathrm{C}_{28} \mathrm{H}_{31} \mathrm{NO}_{6} \mathrm{~S}$ & 65.99 & 6.13 & 18.84 & 65.82 & 6.23 & 18.75 \\
\hline
\end{tabular}

